\title{
Steering Control System About Self Propelled Split Transporter Based On The D-H Method
}

\author{
Wang Xinyu ${ }^{1,2, a}$, Zhao Jingyi ${ }^{1, b}$, Lv Xiaofei ${ }^{3}$ \\ ${ }^{1}$ Hebei Key Laboratory of Heavy Machinery Fluid Power Transmission and Control, Yan Shan \\ University, Qin Huangdao 066004, China; \\ 2Mu Danjiang University, Mu Danjiang 157000, China; \\ 3Xinxing Hebei Engineering and Technology Co. , Ltd, HanDan, HeBei Province, China; \\ awangxinyu@ysu.edu.cn, bzjy@ysu.edu.cn
}

Keywords: The coal mine equipment carrier, Plan steering trajectory, Coordination of steering.

\begin{abstract}
Article take the coal mine equipment carrier for example, integrated use the robot basic theory and electro-hydraulic control system theory to research of planning steering trajectory, coordination and control its speed and so on basic theory. Design the control law, and force through the connecting rod simulation indirect validation. Through the simulation and testing of the connecting rod force indirectly proof its feasibility. For future theoretical study of multi-module and software to connect system to provide a research method.
\end{abstract}

\section{Introduction}

Yan Shan University researched a coordination of the coal mine equipment carrier with a flexible connection of the power part and the carrier part separation, this is taking into account the actual situation of the restricted space in the mine roadway restrictions and Inoue geographical environment ${ }^{[1]}$.

Nonholonomic mobile robots are generally dynamic and unknown work environment, so the nonholonomic mobile robot should have a certain amount of intelligence ${ }^{[2]}$. One of the biggest feature of the nonholonomic mobile robot is its unique ability of the space motion, the direction of motion of the robot only along the tire extended forward. Therefore, does not solve the problem of motion control, no matter what structure are unable to effectively complete a variety of established task.

\section{The Carrier Tracking Trajectory Control Method Based On D-H Method}

The coal mining equipment carrier use the split and flexible connection technology, the intermediate connecting rod connected to reduce the carrier 's turning radius, this design reduces the height of the carrier. The power part and the carrier part are drived by the hydraulic motor, the middle rod is stretched and squeezed only in the starting and stopping moment. Install an encoder on the wheel were used to detect the steering angle and walking distance of the carrier, and respectively install encoder on the two junction points, to detect the angle formed by the two parts, the angle will be involved in track transfer operations and information on the physical location of the two parts ${ }^{[3],[4]}$.

Through the data acquisition and the monitoring system, collect the flexible connection between the two parts of each other positional parameters. Feedback the angle between the rod and the two parts to the controller in order to determine the relative positions of the two parts. Then, according to the acquisition of the power part trajectory, control the carrier part tracking the trajectory of the power parts ${ }^{[5]}$.

First, we need to determine the relative position of the two parts. Established the coordinate systems $\mathrm{O}_{0}-\mathrm{x}_{0} \mathrm{y}_{0}$ and $\mathrm{O}_{3}-\mathrm{x}_{3} \mathrm{y}_{3}$, on the power part reference point $\mathrm{O}_{0}$ points and the carrier part reference point $\mathrm{O}_{3}$ point. Established the coordinate system in the two deputy commissioner of the connecting rod rotation $\mathrm{O}_{1}-\mathrm{x}_{1} \mathrm{y}_{1}$ and $\mathrm{O}_{2}-\mathrm{x}_{2} \mathrm{y}_{2}$, shown in Fig. (1). 


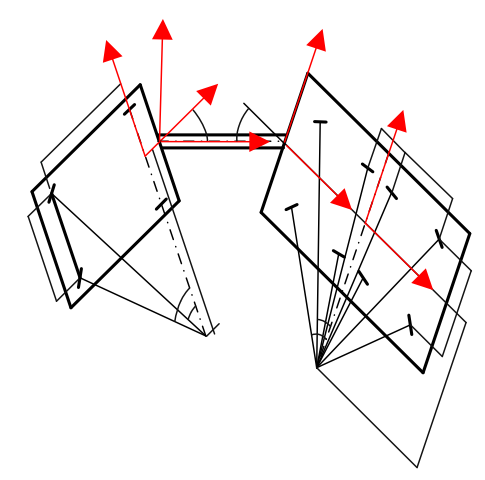

Fig. (1). The model of the coal mine equipment carrier The general expression of the rod transform matrix as follows:

$$
{ }_{i} T=\left[\begin{array}{cccc}
c \theta_{i} & -s \theta_{i} & 0 & a_{i-1} \\
s \theta_{i} c \alpha_{i-1} & c \theta_{i} c \alpha_{i-1} & -s \alpha_{i-1} & -d_{i} s \alpha_{i-1} \\
s \theta_{i} s \alpha_{i-1} & c \theta_{i} s \alpha_{i-1} & c \alpha_{i-1} & d_{i} c \alpha_{i-1} \\
0 & 0 & 0 & 1
\end{array}\right]
$$

Based on the pose transformation equations of the two parts, the carrier part and the power part's position and orientation relationship can be calculated as follows:

$$
\left[\begin{array}{c}
x_{3} \\
y_{3} \\
z_{3} \\
1
\end{array}\right]={ }_{3} T \cdot\left[\begin{array}{c}
x_{0} \\
y_{0} \\
z_{0} \\
1
\end{array}\right]=\left[\begin{array}{cccc}
c(\alpha+\beta) & s(\alpha+\beta) & 0 & b \cdot c(\alpha+\beta)+L \cdot c \alpha+a \\
-s(\alpha+\beta) & c(\alpha+\beta) & 0 & -b \cdot s(\alpha+\beta)-L \cdot s \alpha \\
0 & 0 & 1 & 0 \\
0 & 0 & 0 & 1
\end{array}\right] \cdot\left[\begin{array}{c}
x_{0} \\
y_{0} \\
z_{0} \\
1
\end{array}\right]
$$

Available from the above equation:

$$
\left\{\begin{array}{c}
x_{3}=c(\alpha+\beta) \cdot x_{0}+s(\alpha+\beta) \cdot y_{0}+b \cdot c(\alpha+\beta)+L \cdot c \alpha+a \\
y_{3}=-s(\alpha+\beta) \cdot x_{0}+c(\alpha+\beta) \cdot y_{0}-b \cdot s(\alpha+\beta)-L \cdot s \alpha \\
z_{3}=z_{0}=1
\end{array}\right.
$$

According to the power part's pose and the angle between the connecting rod and the two parts $\alpha$, $\beta$, by equation (3), we can get the position and orientation of the carrier part at this time. Compared the carrier part's position and orientation with the power part's the collected trajectory, according to the above method can be achieved the carrier part's trajectory tracking the power parts', namely the coordination of steering the process of coal mining equipment carrier. We can collect the values of $\alpha, \beta$ in the actual steering of the coal mining equipment carrier ${ }^{[6]}$.

So, the trajectory line of the coal mine equipment carrier can be plan by describe point, and then calculated the speed of two parts to complete the planned path automatically turn in the roadway.

\section{Route Planning}

According to the experimental situation, as Fig. (2), Fig. (3), we assume that the width of the obstacle on the right side of the roadway for the $D_{2}=17500 \mathrm{~mm}$, the initial position of a distance $\mathrm{d} 1$ from the right side wall of the tunnel is $500 \mathrm{~mm}$, and increases the safety distance at high confinement region $d s=150 \mathrm{~mm}$. 

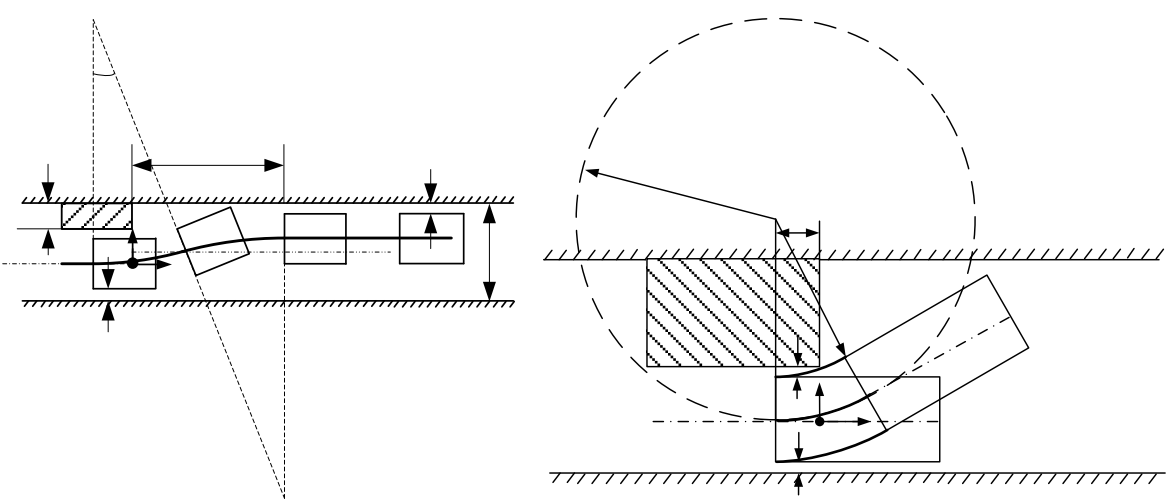

Fig. (2). Schematic of obstacle avoidance trajectory Fig. (3). Process of obstacle avoidance

$\mathrm{S}$ values of the above six groups were generated six groups obstacle avoidance path as shown in Fig. (4). In Fig. (4), three path from left to right represent the left, middle, right side of the first car for the three parts of the reference path.
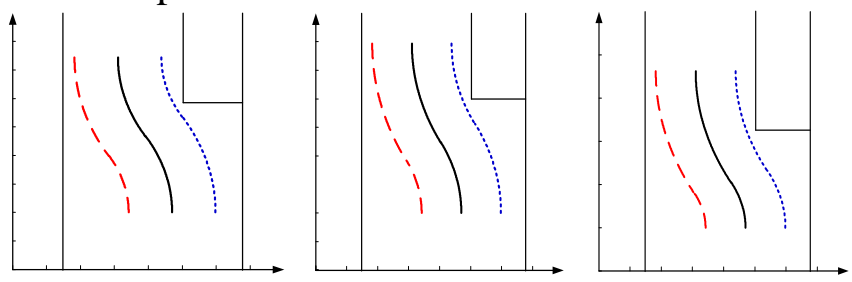

a)s=3.85 m

b)s $=4.00 \mathrm{~m}$

c) $\mathrm{s}=4.50 \mathrm{~m}$
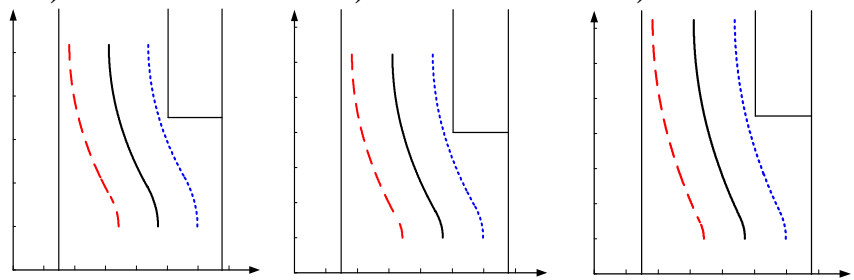

d) $\mathrm{s}=5.00 \mathrm{~m}$

e) $\mathrm{s}=6.00 \mathrm{~m}$

f) $\mathrm{s}=7.00 \mathrm{~m}$

Fig. (4). obstacle avoidance reference path under different $S$

In summary we can see that in the case of a certain conditions, if $\mathrm{s}$ increases, $\beta$ becomes smaller, $\varphi_{2}$ also becomes smaller, this reduce the steering response time.

\section{Experiments}

We placed an obstacle in the side of the corridor, and then control the experimental car through the obstacle sections. Installed hourglass in the same location on the two experimental car use for record the trajectory, Then at a speed of $0.5 \mathrm{~m} / \mathrm{s}$ to complete the obstacle avoidance, And along the trajectory of the travel direction, collect a set of error data every $30 \mathrm{~mm}^{[7],[8]}$. The specific process is shown in Fig. (5).

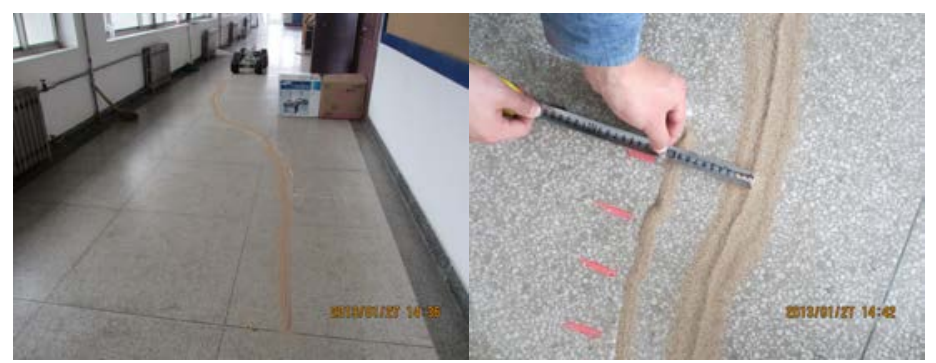

Fig. (5). Actual trajectory

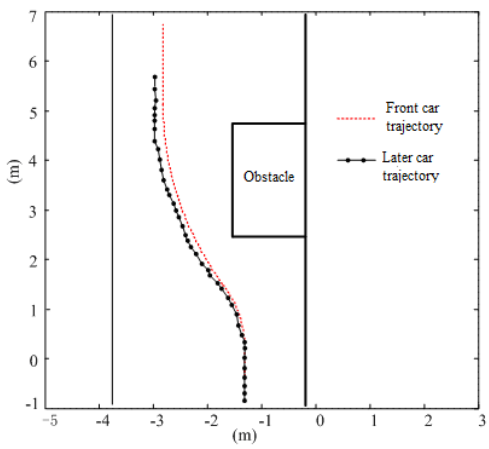

Fig. (6). schematic of the two cartrajectory in obstacle avoidance experiment 
According to the collected data of the two car trajectory to generate the two car trajectory as shown in Fig. (6). During the process of the obstacle avoidance, the error of trajectory is small, this control strategy is similar to steering system characteristics, it have better ability to adapt. But when the length of trajectory and the control action increase, the trajectory error still showed a gradual increasing trend, This trend is mainly due to differences in the basic structure and implementation of the components of the two vehicles caused, which can not be eliminated. So when the driver driving the car, should try to avoid consecutive turns, the control system should be increased vehicular state recognition at the same time, and eliminated error which caused by the action follow the control strategy at any time. When the car are producted and debugged, we should try to make all modules steering system has basically the same dynamic characteristics, Otherwise it will cause greater action following error.

\section{Conclusion And Future Work}

By in-depth exploration and research of the independent steering of the key technologies involved in the system of coal mining equipment carrier. The research results can be generalized to all kinds of Self Propelled Modular Transporter and derivatives such as special loading platform system. This have some reference value for similar products research and development and improvement, have important theoretical significance and application value to improve the domestic truck modernization of installed capacity and production levels.

\section{Acknowledgments}

This research was supported in part by National Natural Science Foundation of China under Grant (No. 51175448); The Open Foundation of the State Key Lab of Fluid Power Transmission and Control(No. GZKF-201103).

\section{References}

[1] Geng Guan-jie. The coal mining equipment carrier electro-hydraulic and key technology practice[D]. [Yanshan University Master of Engineering thesis], (2010) 11-14.

[2] Fangzheng Gao, Jing Xie, Fushun Yuan, "Finite-Time Control of Nonholonomic Mobile Robots", IJACT, Vol. 4, No. 2, pp. 161 168, 2012

[3] Zhao Jing-yi. [The Large Self Propelled Transporter], (2010)

[4] Chen Feng-lei, Zhao Jing-yi, Huang Zi-zhai. Design and development of TMZ80 transporting vehicle for successive coal-mining equipmen, The Seventh International Conference on Fluid Power Transmission and Control (ICFP 2009), Hangzhou, China, 309-312, 2009;

[5] Zhao Mingwang, Wang Jie, Jiang Weihua. Modern control theory [M]. Wuhan: Huazhong University of Science and Technology Press,2007:211-213.

[6] Ren Huirong. The research of the mobile robot tracking control method for Similar vehicles [D] - Tianjin: Journal of Tianjin University control theory and control engineering disciplines,2008:7;

[7] Zhang Chi. [The coal mining equipment carrier electro-hydraulic control system design which based on the CAN bus]. [Yanshan University Master of Engineering thesis], (2010) 64-73.

[8] Fenglei Chen, Jingyi Zhao. Steering Coordinated Control for WC80Y Separate Transporting Platform Based on CAN Bus. 2010 WASE International Conference on Information Engineering (ICIE 2010), (03): 275-278, 2010; 